\title{
Leptophlebiidae ocorrentes no Estado do Rio de Janeiro, Brasil: hábitats, meso-hábitats e hábitos das ninfas (Insecta: Ephemeroptera)
}

\author{
Elidiomar Ribeiro Da-Silva ${ }^{1,3,4}$, Jorge Luiz Nessimian ${ }^{2,3}$ \& Luci Boa Nova Coelho ${ }^{2}$ \\ ${ }^{1}$ Laboratório de Insetos Aquáticos, Departamento de Zoologia, Instituto de Biociências, \\ Universidade Federal do Estado do Rio de Janeiro - UNIRIO, \\ Av. Pasteur, n. 458, $4^{\circ}$ andar, Urca, CEP 22290-240, Rio de Janeiro, RJ, Brasil \\ ${ }^{2}$ Laboratório de Entomologia, Departamento de Zoologia, Instituto de Biologia, \\ Universidade Federal do Rio de Janeiro - UFRJ, CP 68044, CEP 21944-970, Rio de Janeiro, RJ, Brasil, \\ e-mails:nessimia@acd.ufrj.br, lucibncoelho@ufrj.br \\ ${ }^{3}$ Bolsista de Pesquisa do CNPq (http://www.cnpq.br) \\ ${ }^{4}$ Autor para correspondência: Elidiomar Ribeiro Da-Silva, e-mail: elidiomar@pq.cnpq.br
}

DA-SILVA, E.R., NESSIMIAN, J.L. \& COELHO, L.B.N. Leptophlebiidae from Rio de Janeiro State, Brazil: nymphal habitats, mesohabitats, and habits (Insecta: Ephemeroptera). Biota Neotrop. 10(4): http://www. biotaneotropica.org.br/v10n4/en/abstract?article+bn01410042010.

Abstract: The leptophlebiid mayflies from Rio de Janeiro State are still poorly known from faunistic, taxonomic and biological points of view. In this paper were compiled biological data about 14 genera (Askola, Farrodes, Hagenulopsis, Hermanella, Hylister, Leentvaaria, Massartella, Miroculis, Needhamella, Perissophlebiodes, Thraulodes, Traverella, Ulmeritoides, and an undescribed genus) and about 25 morphospecies from Rio de Janeiro. Most of the genera and species of this family are related to rithral habitats, occupying places with deposition of allochtonous organic matter.

Keywords: Atalophlebiinae, mayflies, aquatic insects, ecology, Neotropics.

DA-SILVA, E.R., NESSIMIAN, J.L. \& COELHO, L.B.N. Leptophlebiidae ocorrentes no Estado do Rio de Janeiro, Brasil: hábitats, meso-hábitats e hábitos das ninfas (Insecta: Ephemeroptera). Biota Neotrop. 10(4): http://www.biotaneotropica.org.br/v10n4/pt/abstract?article+bn01410042010.

Resumo: A família Leptophlebiidae é ainda pobremente conhecida no Estado do Rio de Janeiro, tanto em termos faunísticos quanto taxonômicos e biológicos. No presente trabalho foram reunidos dados biológicos acerca de 14 gêneros (Askola, Farrodes, Hagenulopsis, Hermanella, Hylister, Leentvaaria, Massartella, Miroculis, Needhamella, Perissophlebiodes, Thraulodes, Traverella e Ulmeritoides, além de um gênero ainda não descrito) e em torno de 25 morfoespécies ocorrentes no estado. Em termos ecológicos, a maioria dos gêneros e espécies dessa família está associada a ambientes lóticos ritrais, colonizando áreas de deposição de material orgânico alóctone.

Palavras-chave: Atalophlebiinae, efemerópteros, insetos aquáticos, ecologia, região Neotropical. 


\section{Introdução}

O atual estágio de conhecimento dos rios tropicais é ainda deficiente, especialmente no que se refere a seus componentes bióticos (Flowers 1991), dentre os quais se destacam os insetos da ordem Ephemeroptera (Hubbard 1982). Passando a maior parte de seu ciclo de vida como formas aquáticas (imaturos chamados ninfas, náiades ou larvas pelos diferentes autores), as quais vivem em uma grande variedade de hábitats, são elementos biológicos importantes, não somente por sua grande abundância, mas também pelo papel que cumprem no funcionamento dos ecossistemas (Edmunds et al. 1976, Chacón \& Segnini 1996). São componentes importantes do ciclo de nutrientes, devolvendo ao ambiente terrestre (através da respiração e biomassa dos adultos, aéreos), parte das substâncias que foram carreadas para os diferentes corpos de água, o que contribui para manter a qualidade da água. Constituem ainda uma das principais fontes de alimento para peixes, aves e invertebrados, e podem ser utilizados como indicadores das perturbações antropogênicas que afetam os ambientes aquáticos (Chacón \& Segnini 1996).

A família Leptophlebiidae está entre os elementos dominantes dos pequenos rios neotropicais, com mais de cinquenta gêneros descritos (Savage 1987, Domínguez et al. 2001, 2006), 23 deles registrados para o Brasil. Por sua vez, o Estado do Rio de Janeiro tem registrada a ocorrência de 14 gêneros (Da-Silva 2002, Salles 2010, Da-Silva et al. 2010). Deve-se ressalvar que Da-Silva et al. (2010) registraram a ocorrência de nove gêneros, mas com base exclusivamente em espécies nominais. Em realidade, é parco o estágio do conhecimento acerca da fauna de Leptophlebiidae ocorrente no estado, tanto em termos taxonômicos quanto biológicos. A maioria das informações disponíveis acerca de aspectos bioecológicos relacionados ao grupo, dispersa em uns poucos artigos de cunho preponderantemente taxonômico, muitas vezes aparece sob a forma de descrições pouco precisas dos ambientes de criação, hábito das ninfas e, mais raramente, tipo de substrato de ocorrência.

Desde 1986, pesquisadores associados ao Laboratório de Entomologia da Universidade Federal do Rio de Janeiro vêm realizando estudos com os insetos aquáticos do Estado do Rio de Janeiro, visando a elucidação de problemas taxonômicos e biológicos acerca dos diferentes grupos. Como parte desses estudos, o presente artigo objetiva reunir e interpretar as informações publicadas ou originais a respeito dos hábitats e meso-hábitats preferenciais e dos hábitos (modos de existência) das ninfas de Leptophlebiidae ocorrentes no Estado do Rio de Janeiro. Os resultados são expostos seguindo os moldes do trabalho de Carvalho \& Nessimian (1998), sobre insetos da ordem Odonata.

\section{Material e Métodos}

Localizado na Região Sudeste do Brasil, o Estado do Rio de Janeiro ocupa somente cerca de $0,5 \%\left(44.268 \mathrm{~km}^{2}\right)$ do território nacional, sendo um dos menores da União. Não obstante, apresenta grande diversidade fisionômica, reflexo de sua localização litorânea e da presença de inúmeros conjuntos serranos. As composições fisionômicas preponderantes são as restingas, as florestas e os campos de altitude, todos partes da província da Mata Atlântica (Eiten 1992, Carvalho \& Nessimian 1998), de grande diversidade biológica.

De um modo geral, os exemplares estudados foram coligidos em ambientes lóticos, sendo para tal utilizados peneiras e puçás, sempre com malha de, no máximo, 1,0 $\mathrm{mm}$ de abertura, além de amostradores clássicos para insetos aquáticos em geral, como Surber e Hess (Merritt et al. 1996). As coletas foram realizadas nos ambientes mais diversificados, mas que de modo geral se encaixam nas seguintes categorias: ritral alto (acima de $1.000 \mathrm{~m}$ de altitude), ritral baixo e potamal. Procurou-se igualmente registrar nas coletas a distribuição dos indivíduos ao longo dos distintos meso-hábitats de ocorrência, a saber (definições entre parênteses): "folhiço de fundo" (material orgânico alóctone acumulado no leito do rio, em áreas de remanso); "folhiço de superfície" (material orgânico alóctone retido em áreas superficiais de correnteza); "sedimento fino" (areia); "sedimento grosso" (seixos); "matacões" (grandes pedaços de rocha); "vegetação marginal" (áreas marginais, sombreadas, em que partes da vegetação terrestre entram em contato com a água); "hidrófitas" (associação com plantas aquáticas, geralmente em locais de água mais parada).

A classificação dos gêneros de Leptophlebiidae ocorrentes no Estado do Rio de Janeiro quanto à ocupação dos ambientes aquáticos e a seus hábitos seguiu observações realizadas em campo, aliadas a registros constantes na literatura. Hábitats foram classificados com base em Schäfer (1985), Ward (1992), Cummins \& Merritt (1996) e Carvalho \& Nessimian (1998). Embora agrupamentos supraespecíficos não correspondam obrigatoriamente a grupamentos funcionais em termos de ocupação do ambiente (Carvalho \& Nessimian 1988), para a maioria dos Leptophlebiidae a generalização de tais informações no nível de gênero parece pertinente.

Os ambientes de água corrente (hábitats lóticos) foram classificados segundo Schäfer (1985) e Cummins \& Merritt (1996), sendo adotados os termos "ritral" (riachos) e "potamal" (rios). Utilizou-se o termo "semi-lótico" para os ambientes de águas paradas ou lentas resultantes de represamentos de rios ou riachos (Figura 1), à semelhança do utilizado por Carvalho \& Nessimian (1998). A terminologia para a classificação dos hábitos (modos de existência) foi a constante em Cummins \& Merritt (1996), sendo utilizados os termos traduzidos ao português por Carvalho \& Nessimian (1998): "reptantes" (sprawlers), "agarradores" (clingers) e "escaladores" (climbers).

Foram estudados exemplares procedentes dos seguintes municípios (localidades entre parênteses ou colchetes): Angra dos Reis [Bracuí, Caputera, Ilha Grande (Abraão, Dois Rios, Palmas, Praia Preta)], Barra do Piraí (Rio Paraíba do Sul), Barra Mansa (Rio Paraíba do Sul), Cachoeiras de Macacu (Duas Pontes, Japuíba, Santa Mônica), Comendador Levy Gasparian (Mont Serrat), Guapimirim (Parque Nacional da Serra dos Órgãos), Itatiaia (Fazenda Aleluia, Mauá, Parque Nacional do Itatiaia, Rio Paraíba do Sul), Japeri (Santana), Macaé (Sana), Magé (Citrolândia), Mangaratiba (Fazenda Batatal, Reserva Ecológica Rio das Pedras), Maricá (Ubatiba), Miguel Pereira (Conrado), Nova Friburgo (Alto do Cascatinha, Caledônia,

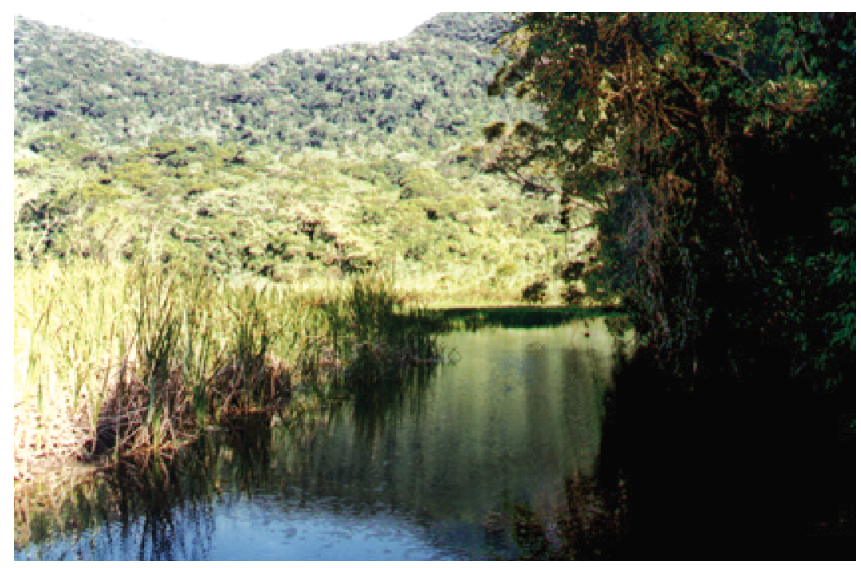

Figura 1. Subaio, Teresópolis, RJ: exemplo de ambiente "semi-lótico". (Foto do acervo do Laboratório de Entomologia da UFRJ.)

Figure 1. Subaio, Teresópolis, RJ: view of a "semi-lotic environment. (Photo from the collection of the Laboratório de Entomologia da UFRJ). 
Cardinot, Cascatinha, Lumiar, Mury, Reserva Ecológica Macaé de Cima, São Pedro da Serra), Parati (Estrada Parati-Cunha, Estrada Parati-Ubatuba), Petrópolis (Correias, Sítio Ribeirão), Pinheiral (Rio Paraíba do Sul), Piraí (Rio Piraí), Resende (Rio Paraíba do Sul), Rio de Janeiro (Maria da Graça, Parque Nacional da Tijuca, Reserva Três Rios, Serra do Mendanha), Teresópolis (Fazenda Vale da Revolta, Granja Guarani, Parque Nacional da Serra dos Órgãos, Represa Guinle, Serrinha, Subaio, Vieira), Volta Redonda (Rio Paraíba do Sul).

Os exemplares foram fixados e conservados em etanol a $80 \%$, estando depositados em quatro instituições, todas localizadas no município do Rio de Janeiro, Estado do Rio de Janeiro, Brasil: Departamento de Biologia, Fundação Instituto Oswaldo Cruz FIOCRUZ; Laboratório de Insetos Aquáticos, Departamento de Zoologia, Instituto de Biociências, Universidade Federal do Estado do Rio de Janeiro - UNIRIO; Coleção Entomológica José Alfredo Pinheiro Dutra, Laboratório de Entomologia, Departamento de Zoologia, Instituto de Biologia, Universidade Federal do Rio de Janeiro - UFRJ; Setor de Insetos Aquáticos, Departamento de Entomologia, Museu Nacional, Universidade Federal do Rio de Janeiro - UFRJ.

\section{Resultados e Discussão}

As fontes de informação utilizadas para o presente estudo estão agrupadas na Tabela 1. No total, foram reunidos dados biológicos de quatorze gêneros e em torno de 25 morfoespécies de Leptophlebiidae, sendo dezessete identificadas (incluindo espécies ainda não descritas): Askola froehlichi Peters, 1969, Farrodes carioca Dominguez, Molineri \& Peters, 1996 (Figura 2), Hylister plaumanni Dominguez \& Flowers, 1989 (Figura 3), Massartella alegrettae Ulmer, 1943, M. brieni (Lestage, 1924), Miroculis froehlichi Savage \& Peters, 1983 (Figura 4), Perissophlebiodes flinti (Savage, 1982), Thraulodes itatiajanus Traver \& Edmunds, 1967 (Figura 5), além de espécies não descritas de Hermanella Needham \& Murphy, 1924, Leentvaaria

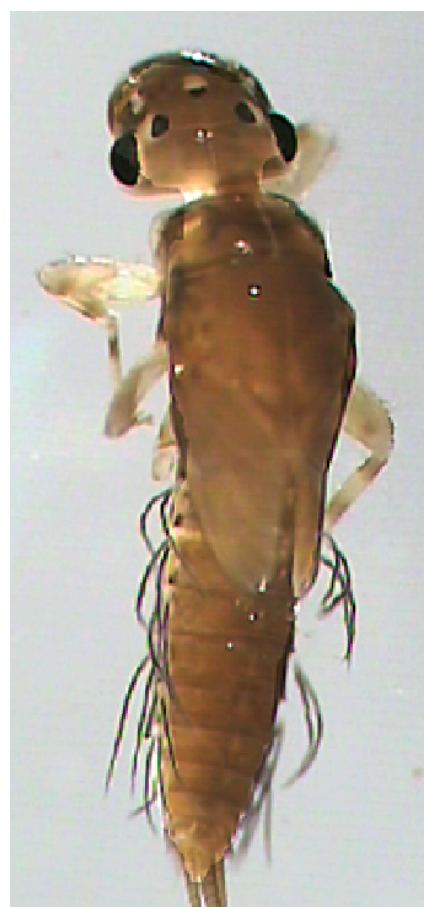

Figura 2. Farrodes carioca, ninfa madura.

Figure 2. Farrodes carioca, mature nymph.

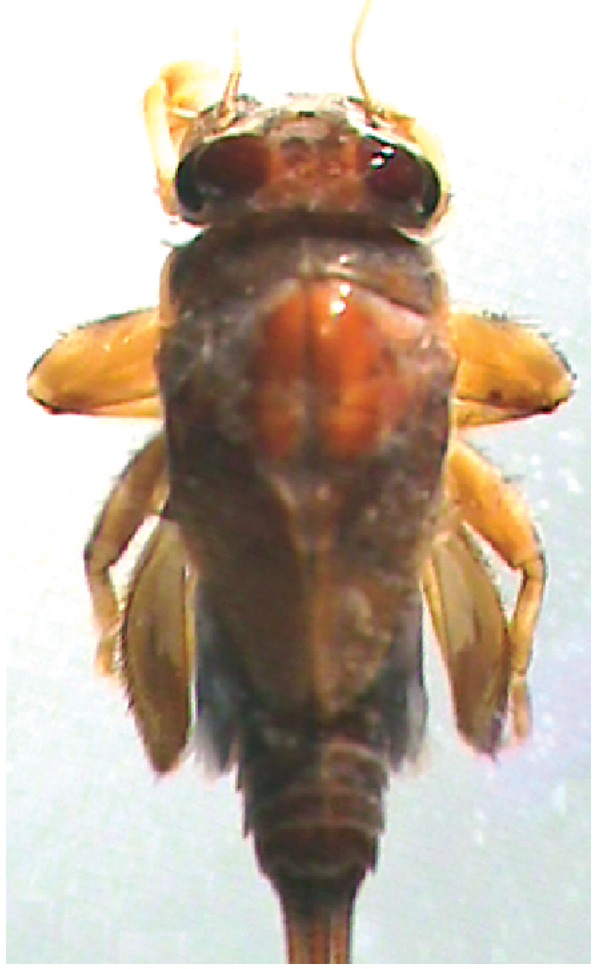

Figura 3. Hylister plaumanni, ninfa madura.

Figure 3. Hylister plaumanni, mature nymph.

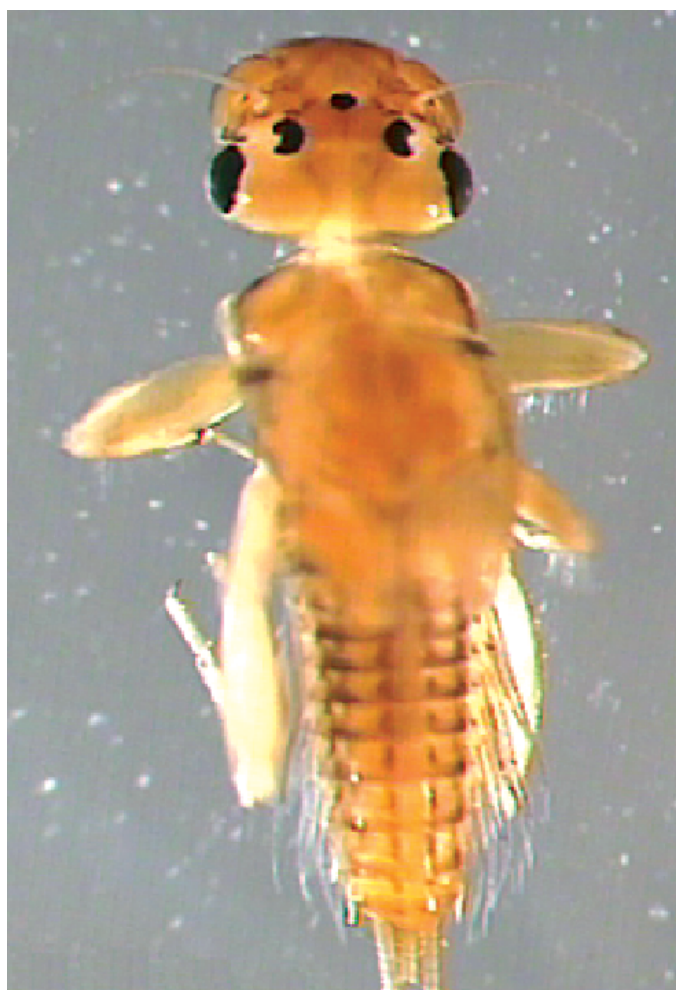

Figura 4. Miroculis froehlichi, ninfa madura.

Figure 4. Miroculis froehlichi, mature nymph. 
Tabela 1. Fontes de informação sobre preferência de hábitats para criação e hábitos (modos de existência) das ninfas de Leptophlebiidae ocorrentes no Estado do Rio de Janeiro, utilizadas no presente estudo. Registros assinalados com asterisco são originais (registros assinalados para espécies não determinadas referem-se ao gênero). Informações obtidas acerca de espécies não registradas em território fluminense, mas pertencentes a gêneros aqui ocorrentes, foram incluídas.

Table 1. Sources of information about habitat preference for rearing and habits (modes of existence) of nymphs of Leptophlebiidae from Rio de Janeiro State. Original records are marked with an asterisk (records marked for unidentified are refered to genus). Information about species not recorded in Rio de Janeiro but belonging to genera occuring here were included.

\begin{tabular}{lll}
\hline \multicolumn{1}{c}{ Táxons } & \multicolumn{1}{c}{ Registros observados } & \multicolumn{1}{c}{ Referências } \\
\hline Askola froehlichi Peters, 1969 & $\begin{array}{l}\text { Angra dos Reis, Itatiaia, Nova Friburgo, Da-Silva (1997, 2002), Baptista et al. } \\
\text { Teresópolis } \\
\text { Farrodes Peters, 1971 }\end{array}$ & \multicolumn{1}{c}{$\begin{array}{l}\text { Lugo-Ortiz \& McCafferty (1994) } \\
\text { Farrodes carioca Domínguez, }\end{array}$} \\
Molineri \& Peters, 1996 & $\begin{array}{l}\text { Comendador Levy Gasparian, Itatiaia, Macaé, Da-Silva (2002), Andrade et al. (2003) } \\
\text { Farrodes spp. }\end{array}$ & $\begin{array}{l}\text { Mangaratiba, Nova Friburgo, Teresópolis } \\
\text { Barra do Piraí*, Barra Mansa*, Cachoeiras de Baptista et al. (1998a,b) } \\
\text { Macacu*, Casimiro de Abreu, Itatiaia, Miguel }\end{array}$ \\
& $\begin{array}{l}\text { Pereira*, Nova Friburgo, Pinheiral*, Volta } \\
\text { Redonda* }\end{array}$
\end{tabular}

Hagenulopsis Ulmer, 1920

Hagenulopsis spp.

Hermanella Needham \& Murphy, 1924

Hermanella sp., espécie nova

Hermanella sp.

Hylister plaumanni Domínguez \& Flowers, 1989

Leentvaaria Demoulin, 1966

Leentvaaria sp., espécie nova

Massartella Lestage, 1930

Massartella alegrettae Ulmer, 1943

Massartella brieni (Lestage, 1924)

Massartella sp. 1, espécie nova

Massartella sp. 2, espécie nova

Massartella spp.

Miroculis Edmunds, 1963

Miroculis froehlichi Savage \& Peters, 1983

Miroculis sp.

Needhamella Domínguez \& Flowers, 1989

Needhamella sp., espécie nova

Needhamella sp.

Perissophlebiodes flinti (Savage, 1982)
Hofmann et al. (1999)

Casimiro de Abreu, Parati*, Petrópolis*, Rio Baptista et al. (1998a, b) de Janeiro*

Domínguez \& Flowers (1989), Ferreira \& Domínguez (1992)

Itatiaia

Da-Silva (2002)

Itatiaia, Teresópolis*

Cachoeiras de Macacu, Nova Friburgo, Petrópolis, Teresópolis

Domínguez \& Flowers (1989), DaSilva \& Pereira (1993), Da-Silva (1997, 2002), Baptista et al. (1998b), Andrade et al. 2003

Demoulin (1966), Lopes (1999)

Comendador Levy Gasparian

Da-Silva (2002)

Pescador \& Peters (1990)

Teresópolis Da-Silva (2002)

Angra dos Reis, Itatiaia, Mangaratiba, Nova Pescador \& Peters (1990), Friburgo, Rio de Janeiro, Teresópolis Baptista et al. (1998b), Da-Silva (2002), Andrade et al. (2003)

Nova Friburgo

Da-Silva (2002)

Nova Friburgo

Da-Silva (2002)

Angra dos Reis, Itatiaia

Savage \& Peters (1983)

Angra dos Reis, Cachoeiras de Macacu, Da-Silva (1997, 2002), Baptista et al. Casimiro de Abreu, Comendador Levy (1998a, b), Andrade et al. (2003) Gasparian, Itatiaia, Macaé, Mangaratiba, Nova Friburgo, Rio de Janeiro, Teresópolis

Magé*, Maricá*, Miguel Pereira*, Nova Baptista et al. (1998a,b) Friburgo

Domínguez \& Flowers (1989)

Comendador Levy Gasparian Da-Silva (2002)

Piraí Da-Silva (1997)

Nova Friburgo Savage (1982) 
Tabela 1. Continuação...

\begin{tabular}{|c|c|c|}
\hline Táxons & Registros observados & Referências \\
\hline Thraulodes Ulmer, 1920 & - & $\begin{array}{l}\text { Traver (1944); Traver \& Edmunds } \\
\text { (1967), Allen \& Brusca (1978), } \\
\text { Domínguez (1986, 1987), Ferreira \& } \\
\text { Froehlich (1992), Chacón et al. (1999), } \\
\text { Callisto \& Goulart (2000), Lopes et al. } \\
\text { (2003) }\end{array}$ \\
\hline
\end{tabular}

Thraulodes itatiajanus Traver \& Edmunds, 1967

Thraulodes sp.

Thraulodes spp.

Traverella Edmunds, 1948

Traverella sp., espécie nova

Ulmeritoides Traver, 1959

Ulmeritoides sp., espécie nova

Ulmeritoides spp.

Atalophlebiinae gen. A sp., gênero novo e espécie nova

Angra dos Reis, Itatiaia, Mangaratiba, Nova Da-Silva (2002)

Friburgo, Parati, Teresópolis

Itatiaia

Da-Silva (2002)

Miguel Pereira*, Nova Friburgo, Petrópolis*, Baptista et al. (1998b)

Rio de Janeiro*

$-$

Comendador Levy Gasparian

-

Teresópolis

Angra dos Reis*, Mangaratiba*, Nova Friburgo, Baptista et al. (1998a, b)

Teresópolis

Allen (1973), Epler (1986), Lugo-Ortiz \& McCafferty (1996)

Da-Silva (2002)

Demoulin (1955), Domínguez (1995), Lopes \& Da-Silva (2003)

Da-Silva (2002)

Teresópolis

Da-Silva (2002)
Demoulin, 1966, Massartella Lestage, 1930, Needhamella Dominguez \& Flowers, 1989, Thraulodes Ulmer, 1920, Traverella Edmunds, 1948, Ulmeritoides Traver, 1959 e de um gênero novo.

Informações sobre gêneros e espécies ocorrentes no Estado do Rio de Janeiro obtidas de fontes que utilizaram material de localidades de outros estados ou países também foram levadas em consideração. Algumas exemplares não foram identificados até o nível específico devido ao precário estado do material (com danos morfológicos) ou ao fato de estarem em estádios muito precoces do desenvolvimento (ninfas muito jovens).

A partir do exame das fontes citadas na Tabela 1 e de informações inéditas, constituiu-se a Tabela 2 , que reúne os principais resultados deste estudo, contendo as informações acerca dos hábitats e mesohábitats de ocorrência e os hábitos das ninfas dos gêneros de Leptophlebiidae. Alguns táxons merecem certo destaque. Farrodes carioca (Figura 2) e Miroculis froehlichi (Figura 4), por exemplo, ocorrem tanto em ambientes ritrais quanto potamais (Da-Silva 2002), e até mesmo em áreas de represamento. Essa última espécie, por sinal, está entre os Ephemeroptera mais encontradiços em corpos lóticos do Estado do Rio de Janeiro. Os gêneros Leentvaaria, Needhamella e Traverella, observados aqui ocorrendo em ambientes potamais (cf. Da-Silva 2002), estão registrados em outras localidades habitando corpos de água ritrais (Domínguez et al. 2006).

Além dos já citados Farrodes Peters, 1971 e Miroculis Edmunds, 1963, outros gêneros podem ocupar ambientes semi-lóticos, como Thraulodes e Ulmeritoides. Ulmeritoides, por sinal, parece ter preferência por hábitats de águas mais paradas, como brejos associados a ritrais. A estrutura de suas brânquias (Da-Silva 2002, Da-Silva et al. 2002), com grande quantidade de filamentos, pode representar uma adaptação para facilitar a existência em locais menos oxigenados.

Em relação à Massartella, esse parece ser um dos poucos Leptophlebiidae em que generalizações sobre locais de ocupação podem ser enganosas. O gênero parece estar relacionado a áreas de acúmulo de folhiço, tanto de fundo quanto de superfície. Entretanto, uma espécie, ainda não descrita - Massartella sp. 1 - foi observada também, com certa frequência, deslocando-se habilmente entre os seixos de um trecho correntoso do rio (Rio Cascatinha, localidade Alto do Cascatinha, trecho aqui classificado como ritral alto), o que caracteriza o meso-hábitat sedimento grosso. Em termos morfológicos, tal espécie é mais achatada dorsoventralmente que as demais, apresentando ainda o pronoto mais expandido lateralmente (Da-Silva 2002), o que poderia facilitar a vida nesse substrato. Além disso, enquanto as outras espécies de Massartella têm seis ou sete pares de brânquias abdominais, Massartella sp. 1 apresenta apenas cinco pares, possível adaptação à vida em locais de maior correnteza.

Quanto à Perissophlebiodes Savage, 1983, sua classificação em termos de meso-hábitat e hábitos é estimada. Não foram observados exemplares para a realização do presente trabalho, sendo as informações inferidas a partir da descrição da única espécie do gênero, P. flinti (Savage 1982). Tal espécie apresenta garras tarsais com dentículos medianos bem desenvolvidos e brânquias muito delgadas, características que poderiam facilitar a existência em hábitats de forte correnteza, o que, aliás, é o caso de sua localidade-tipo.

Em termos de hábitos, de um modo geral a maioria dos Leptophlebiidae associados a meso-hábitats em áreas de deposição (como folhiço e sedimento fino) tem hábitos reptantes. Os que vivem em áreas de maior correnteza são agarradores, ainda que possam também apresentar hábito reptante. Finalmente, Ulmeritoides, o único gênero observado com aparente preferência por hábitats semi-lóticos, pode atuar como escalador em hidrófitas ou no folhiço.

Mesmo tendo sido aqui registrados cerca de $60 \%$ dos gêneros de Leptophlebiidae ocorrentes no Brasil, o estudo da família no estado deve ser considerado em estágio ainda preliminar. Para um diagnóstico mais abrangente do grupo são necessárias ainda mais coletas e observações, especialmente em regiões mais ao norte do estado, ressalvando-se que as áreas em que foram obtidos exemplares para esse estudo são possivelmente as mais representativas no que 
Tabela 2. Classificação preliminar de hábitats, meso-hábitats e hábitos das ninfas de Leptophlebiidae do Estado do Rio de Janeiro, baseada em observações de campo realizadas e nas fontes citadas na Tabela 1. A ordenação adotada para a disposição das informações indica provável ordem de preferência. Ponto de interrogação (?) indica classificação estimada. Legenda: * - sobre folhiço; ** - sob seixos; *** - sobre matacões.

Table 2. Preliminary classification of habitats, meso-habitats, and habits of leptophlebiids from Rio de Janeiro State, based on field observations and the sources cited in Table I. The ordering adopted for the provided information indicates a probable order of preference. Question mark (?) indicates estimated classification. Legend: * - on litter, ** - under pebbles; *** - on boulders.

\begin{tabular}{|c|c|c|c|}
\hline Gênero & Hábitat & Meso-hábitat & Hábito \\
\hline Askola & Lótico: ritral alto & Sedimento fino; folhiço de fundo & Reptante*. \\
\hline Farrodes & Lótico: ritral baixo; potamal & Folhiço de fundo; vegetação marginal & Reptante*; escalador. \\
\hline Hagenulopsis & Lótico: ritral baixo & Folhiço de superfície & Reptante*. \\
\hline Hermanella & Lótico: ritral baixo & Folhiço de fundo; sedimento grosso & $\begin{array}{l}\text { Reptante*; reptante**; } \\
\text { agarrador. }\end{array}$ \\
\hline Hylister & Lótico: ritral baixo; ritral alto & Sedimento grosso; folhiço de fundo & $\begin{array}{l}\text { Reptante**; agarrador; } \\
\text { reptante*. }\end{array}$ \\
\hline Leentvaaria & Lótico: potamal; ritral baixo & Sobre matacões & Reptante $* * *$; agarrador. \\
\hline Massartella & $\begin{array}{l}\text { Lótico: ritral alto; ritral baixo } \\
\text { Semi-lótico: represamentos de ritral }\end{array}$ & $\begin{array}{l}\text { Folhiço de fundo; folhiço de superfície, } \\
\text { sedimento grosso }\end{array}$ & $\begin{array}{l}\text { Reptante*; reptante**; } \\
\text { agarrador. }\end{array}$ \\
\hline Miroculis & $\begin{array}{l}\text { Lótico: ritral baixo; ritral alto; potamal } \\
\text { Semi-lótico: represamentos de ritral }\end{array}$ & $\begin{array}{l}\text { Folhiço de fundo; folhiço de superfície; } \\
\text { sedimento fino }\end{array}$ & Reptante*; reptante**. \\
\hline Needhamella & Lótico: potamal; ritral baixo & $\begin{array}{l}\text { Sobre matacões; edimento grosso; } \\
\text { sedimento fino }\end{array}$ & $\begin{array}{l}\text { Reptante***; reptante**; } \\
\text { agarrador. }\end{array}$ \\
\hline Perissophlebiodes & Lótico: ritral baixo & Sedimento grosso? & Reptante**?; agarrador? \\
\hline Thraulodes & $\begin{array}{l}\text { Lótico: ritral baixo } \\
\text { Semi-lótico: represamentos de ritral }\end{array}$ & Sedimento grosso; folhiço de fundo & Reptante**; agarrador. \\
\hline Traverella & Lótico: potamal; ritral baixo & Sobre matacões; sedimento grosso & Reptante ${ }^{* * *}$; agarrador. \\
\hline Ulmeritoides & $\begin{array}{l}\text { Semi-lótico: represamentos de ritral; brejos } \\
\text { de altitude } \\
\text { Lótico: ritral alto; ritral baixo }\end{array}$ & $\begin{array}{l}\text { Folhiço de fundo; folhiço de superfície; } \\
\text { hidrófitas }\end{array}$ & Escalador; reptante* \\
\hline $\begin{array}{l}\text { Atalophlebiinae gen. A, } \\
\text { gênero novo }\end{array}$ & Lótico: ritral baixo & Sedimento grosso & Reptante** \\
\hline
\end{tabular}

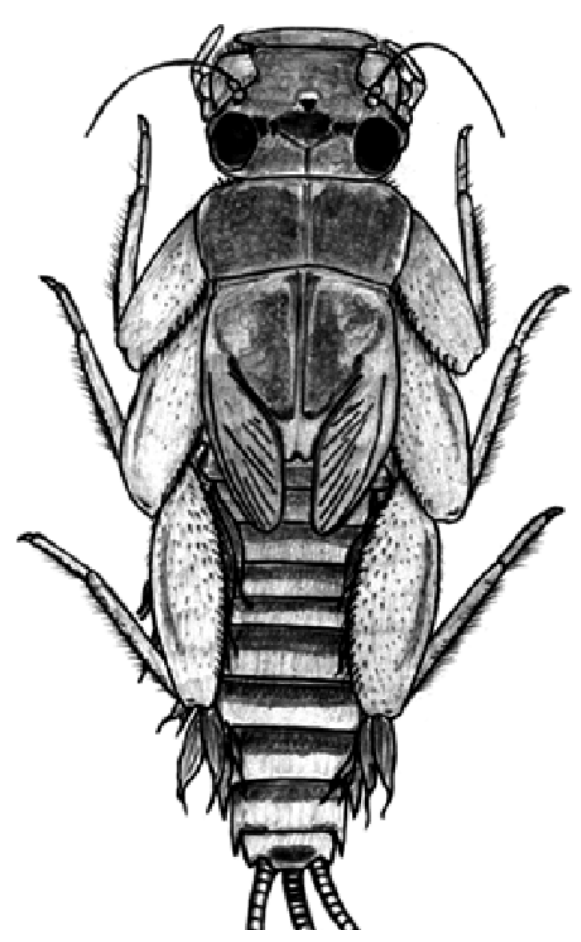

Figura 5. Thraulodes itatiajanus, ninfa madura.

Figure 5. Thraulodes itatiajanus, mature nymph. se refere aos hábitats preferenciais de ninfas de Leptophlebiidae (Hubbard 1990), os ambientes lóticos ritrais de pequeno a médio porte. Ainda assim, não será surpreendente se novos Leptophlebiidae vierem a ser, brevemente, acrescidos à fauna fluminense, o que, aliás, é fato concreto para a maioria dos grupos zoológicos.

\section{Agradecimentos}

Ao Conselho Nacional de Desenvolvimento Científico e Tecnológico $(\mathrm{CNPq})$ e à Fundação de Amparo à Pesquisa no Estado do Rio de Janeiro (FAPERJ), pelo auxílio financeiro.

\section{Referências Bibliográficas}

ALLEN, R.K. \& BRUSCA, R.C. 1978. Generic revisions of mayfly nymphs II. Thraulodes in North and Central America (Leptophlebiidae). Can. Ent. 110:413-433.

ALLEN, R.K. 1973. Generic revisions of mayfly nymphs. 1. Traverella in North and Central America (Leptophlebiidae). Ann. Ent. Soc. Am. 66:1287-1295.

ANDRADE, M.B., SICILIANO, A.T., DA-SILVA, E.R. \& SALLES, F.F. 2003. A efemeropterofauna do Rio Paquequer, Parque Nacional da Serra dos Órgãos, Teresópolis, RJ: composição preliminar e mesodistribuição (Insecta: Ephemeroptera). In Publicações científicas da UNIRIO (A.M.B. Carvalho \& D.M. Silva-Matos, ed.). Universidade Federal do Estado do Rio de Janeiro, Rio de Janeiro, p.1-6. 1 CD-ROM.

BAPTISTA, D.F., DORVILLÉ, L.F.M., BUSS, D.F. \& NESSIMIAN, J.L. 1998a. Distribuição de comunidades de insetos aquáticos no gradiente longitudinal de uma bacia fluvial do sudeste brasileiro. In Ecologia de insetos aquáticos (J.L. Nessimian \& A.L. Carvalho, ed.). Universidade Federal do Rio de Janeiro, Rio de Janeiro, p.191-207. Series Oecologia Brasiliensis, V. 
BAPTISTA, D.F., BUSS, D.F., DORVILLÉ, L.F.M. \& NESSIMIAN, J.L. 1998b. O Conceito de Continuidade de Rios é válido para rios de mata atlântica do sudeste do Brasil? In Ecologia de insetos aquáticos (J.L. Nessimian \& A.L. Carvalho, ed.). Universidade Federal do Rio de Janeiro, Rio de Janeiro, p.209-222. Series Oecologia Brasiliensis, V.

CALLISTO, M. \& GOULART, M.D.C. 2000. Phoretic association between Nanocladius (Plecopteracoluthus) sp. (Chironomidae: Diptera) and Thraulodes sp. (Leptophlebiidae: Ephemeroptera). An. Soc. Ent. Bras. 29(3):605-608

CARVALHO, A.L. \& NESSIMIAN, J.L. 1998. Odonata do Estado do Rio de Janeiro, Brasil: hábitats e hábitos das larvas. In Ecologia de insetos aquáticos (J.L. Nessimian \& A.L. Carvalho, ed.). Universidade Federal do Rio de Janeiro, Rio de Janeiro, p.3-28. Series Oecologia Brasiliensis, V.

CHACÓN, M.M. \& SEGNINI, S. 1996. Reconocimiento taxonomico de las nayades del orden Ephemeroptera en la deriva de dos rios de alta montaña en el Estado Mérida, Venezuela. Bol. Ent. Venez. 11(2):103-122.

CHACÓN, M.M., SEGNINI, S. \& DOMÍNGUEZ, E. 1999. Three new species of Thraulodes (Ephemeroptera: Leptophlebiidae: Atalophlebiinae) from Venezuela. Aquat. Insects 21(4):249-257.

CUMMINS, K.W. \& MERRITT, R.W. 1996. Ecology and distribution of aquatic insects. In An introduction to the aquatic insects of North America. 3 ed. (R.W. Merritt \& K.W. Cummins, ed.). Kendall/Hunt, Dubuque, p.74-86.

DA-SILVA, E.R. \& PEREIRA, S.M. 1993. Efemerópteros da Serra dos Órgãos, Estado do Rio de Janeiro. III. Descrição de uma nova espécie de Lachlania Hagen, 1868 (Ephemeroptera: Oligoneuriidae). An. Acad. Bras. Ci. 65:295-301.

DA-SILVA, E.R. 1997. New and additional records of Leptophlebiidae (Ephemeroptera) from Rio de Janeiro State, Brazil. Rev. Biol. Trop. 44(3)/45(1):684-685.

DA-SILVA, E.R. 2002. Leptophlebiidae (Insecta: Ephemeroptera) ocorrentes no Estado do Rio de Janeiro: taxonomia e caracterização biológica das ninfas. Tese de doutorado, Universidade Federal do Rio de Janeiro, Rio de Janeiro.

DA-SILVA, E.R., GONÇALVES, I.C. \& DE-SOUZA, M.R. 2010. Lista de espécies da ordem Ephemeroptera (Insecta) ocorrentes no Estado do Rio de Janeiro, Brasil. Arq. Mus. Nac. 67(3-4):383-394.

DA-SILVA, E.R., SALLES, F.F. \& BAPTISTA, M.S. 2002. As brânquias dos gêneros de Leptophlebiidae (Insecta: Ephemeroptera) ocorrentes no Estado do Rio de Janeiro. Biota Neotrop. 2(2):1-4.

DEMOULIN, G. 1955. Une mission biologique belge au Brésil. Éphéméroptères. Bull. Inst. R. Sci. Nat. Belg. 31(20):1-32.

DEMOULIN, G. 1966. Contribution a l'etude des Éphéméroptères du Surinam. Bull. Inst. R. Sci. Nat. Belg. 42:1-22.

DOMÍNGUEZ, E. \& FLOWERS, R.W. 1989. A revision of Hermanella and related genera (Ephemeroptera: Leptophlebiidae: Atalophlebiinae) from subtropical South America. Ann. Ent. Soc. Am. 82:555-573.

DOMÍNGUEZ, E. 1986. Thraulodes bolivianus, una nueva especie de la familia Leptophlebiidae (Insecta: Ephemeroptera) de Bolivia. Acta Zool. Lilloana 38(2):149-153.

DOMÍNGUEZ,E. 1987. El género Thraulodes (Ephemeroptera:Leptophlebiidae) en la República Argentina. Acta Zool. Lilloana 39:47-65.

DOMÍNGUEZ, E. 1995. Cladistic analysis of the Ulmeritus-Ulmeritoides group (Ephemeroptera, Leptophlebiidae), with descriptions of five new species of Ulmeritoides. J. N. York Ent. Soc. 103(1):15-38.

DOMÍNGUEZ, E., HUBBARD, M.D., PESCADOR, M.L. \& MOLINERI, C. 2001. Checklist of the Ephemeroptera of South America. http://www. famu.org/mayfly/sacat.html (último acesso em 20/06/2003).

DOMINGUEZ, E., MOLINERI, C., PESCADOR, M.L., HUBBARD, M.D. \& NIETO, C. 2006. Ephemeroptera of South America. In Aquatic biodiversity in Latin America (J. Adis, J.R. Arias, G. Rueda-Delgado \& K.M. Wantzen, ed.). Pensoft, Sofia, p.1-646.

EDMUNDS, G.F., JENSEN, S.L. \& BERNER, L. 1976. The mayflies of North and Central America. University of Minnesota Press, Minneapolis.

EITEN, G. 1992. Natural Brazilian vegetation types and their causes. An. Acad. Bras. Ci. 64(Supl. 1):35-65.
EPLER, J.H. 1986. A novel new neotropical Nanocladius (Diptera Chironomidae), symphoretic on Traverella (Ephemeroptera: Leptophlebiidae). Florida Ent. 69:319-327.

FERREIRA, M.J.N. \& DOMÍNGUEZ, E. 1992. A new species of Hermanella (Ephemeroptera: Leptophlebiidae: Atalophlebiinae) from Southeastern Brazil. Aquat. Insects 14(3):179-182.

FERREIRA, M.J.N. \& FROEHLICH, C.G. 1992. Estudo da fauna de Ephemeroptera (Insecta) do Córrego do Pedregulho (Pedregulho, SP, Brasil) com aspectos da biologia de Thraulodes schilingeri Traver \& Edmunds, 1967. Rev. Bras. Ent. 36(3):541-548.

FLOWERS, R.W. 1991. Diversity of stream-living insects in northwestern Panama. J. N. Am. Benthol. Soc. 10(3):322-334.

HOFMANN, C., SARTORI, M. \& THOMAS, A. 1999. Les Ephéméroptères (Ephemeroptera) de la Guadeloupe (petites Antilles françaises). Mem. Soc. Vaud. Sci. Nat. 20(1):1-95.

HUBBARD, M.D. 1982. Catálogo abreviado de Ephemeroptera da América do Sul. Papéis Avul. Zool. 34(24):257-282.

HUBBARD, M.D. 1990. Mayflies of the World. A catalog of the family and genus group taxa (Insecta: Ephemeroptera). Sandhill, Gainsville. Flora \& Fauna Handbook, 8.

LOPES, M.J.N. \& DA-SILVA, E.R. 2003. A new species of Ulmeritoides from Brazil (Ephemeroptera: Leptophlebiidae). Rev. Biol. Trop. 51(1):195-200

LOPES, M.J.N. 1999. Sistemática de Atalophlebiinae (Insecta: Ephemeroptera, Leptophlebiidae) nos escudos das Guianas e Brasileiro (Rondônia). Tese de Doutorado, Instituto Nacional de Pesquisas da Amazônia, Universidade do Amazonas, Manaus.

LOPES, M.J.N., FROEHLICH, C.G. \& DOMÍNGUEZ, E. 2003. Description of the larva of Thraulodes schlingeri (Ephemeroptera, Leptophlebiidae). Iheringia, Sér. Zool. 93(2):197-200.

LUGO-ORTIZ, C.R. \& McCAFFERTY, W.P. 1994. Farrodes (Ephemeroptera: Leptophlebiidae) in the Antilles: new species from Puerto Rico and review of the genus. Ent. News 105:263-266.

LUGO-ORTIZ, C.R. \& McCAFFERTY, W.P. 1996. New species of Leptophlebiidae (Ephemeroptera) from Mexico and Central America. Ann. Limnol. 32:3-18.

MERRITT, R.W., RESH, V.H. \& CUMMINS, K.W. 1996. Design of aquatic insects studies: collecting, sampling and rearing procedures. In An introduction to the aquatic insects of North America. 3 ed. (R.W. Merritt \& K.W. Cummins, ed.). Kendall/Hunt, Dubuque, p.12-28.

PESCADOR, M.L. \& PETERS, W.L. 1990. Biosystematics of the genus Massartella Lestage (Ephemeroptera: Leptophlebiidae: Atalophlebiinae) from South America. Aquat. Insects 12:145-160.

SALLES, F.F. 2010. Ephemeroptera do Brasil. http://ephemeroptera. br.googlepages.com/home (último acesso em 09/08/2010).

SAVAGE, H.M. \& PETERS, W.L. 1983. Systematics of Miroculis and related genera from northern South America (Ephemeroptera: Leptophlebiidae). Trans. Am. Ent. Soc. 108:491-600.

SAVAGE, H.M. 1982. A curious new genus and species of Atalophlebiinae (Ephemeroptera: Leptophlebiidae) from the Southern coastal mountains of Brazil. Stud. Neotr. Fauna Environ. 17:209-217.

SAVAGE, H.M. 1987. Biogeographic classification of the Neotropical Leptophlebiidae (Ephemeroptera) based uppon geological centers of ancestral origin and ecology. Stud. Neotr. Fauna Environ. 22(4):199-222.

SCHÄFER, A. 1985. Fundamentos de ecologia e biogeografia das águas continentais. Editora da Universidade Federal do Rio Grande do Sul, Porto Alegre.

TRAVER, J.R. \& EDMUNDS, G.F. 1967. A revision of the genus Thraulodes (Ephemeroptera: Leptophlebiidae). Misc. Publ. Ent. Soc. Am. 5:349-404.

TRAVER, J.R. 1944. Notes on Brazilian mayflies. Bolm. Mus. Nac. 22:1-53.

WARD, J.V. 1992. Aquatic insect ecology. 1. Biology and habitat. Wiley, New York. 
